\title{
A new physiological method for heart rate correction of the QT interval
}

P Davey

\begin{abstract}
Aim-To reassess QT interval rate correction.

Background-The QT interval is strongly and inversely related to heart rate. To compare QT intervals between different subjects with different heart rates requires the application of a QT interval rate correction formula. To date these formulae have inappropriately assumed a fixed relation between $Q T$ interval and heart rate. An alternative method of $Q T$ interval rate correction that makes no assumptions about the QT interval-heart rate relation is needed.

Proposal-A QT heart rate correction method should maintain or accentuate biological QT interval variability, should totally remove the dependence of the rate corrected QT interval on heart rate, and should be applicable over a wide range of conditions with a wide range of differing autonomic states.

Methods-QT intervals were obtained at rest and during exercise from subjects expected to have different QT intervals and different $Q T$ interval-heart rate relations. A linear regression line was obtained from the exercise test data, and the QT interval at a notional heart rate of 60 and 0 beats/min, termed the $\mathbf{Q T}{ }_{60}$ interval, and the QT y intercept obtained by back calculation.

Results-QT ${ }_{60}$ and $\mathbf{Q T}$ y intercept values were prolonged in heart failure compared with either left ventricular hypertrophy or controls. There was no relation between heart rate and either $\mathbf{Q T}_{60}$ or $\mathrm{QT} y$ intercept

Conclusions-This new physiologically based method of correcting QT interval for heart rate removes the dependence of the corrected QT interval on heart rate, and maintains biological differences.

(Heart 1999;82:183-186)
\end{abstract}

Keywords: QT interval; heart rate correction

Department of Cardiovascular

Medicine, Level 2, John Radcliffe Hospital, Oxford OX3 9DU, UK P Davey

Correspondence to: Dr Davey.

email: patrick.davey@ ndm.ox.ac.uk

Accepted for publication 3 February 1999

The QT interval is increasingly often measured, partly to assess proarrhythmia in clinical practice, partly to determine the cardiac toxicity of non-cardiac drugs, and partly in the assessment of new antiarrhythmic agents. As heart rate has a powerful influence on the duration of the QT interval, to compare the QT interval from different subjects with different heart rates the QT interval needs to be heart rate corrected to a notional and identical heart rate. Various QT interval heart rate correction formulae have been proposed..$^{1-9}$ There are two basic tests of the validity of the QT interval rate correction formulae. These tests are first, that the dependence of QT interval on heart rate should be totally removed by the application of the rate correction formula, and second, that the QT rate correction formulae should maintain or accentuate genuine biological differences in the QT interval between subjects. The formulae proposed to date have not been totally successful, principally in that corrected QT intervals still show rate dependence. Thus a new approach to QT interval rate correction is needed.

The basic assumption of rate correction formulae is that there is a fixed relation between QT interval and heart rate- - that is, knowledge of the QT interval at one heart rate automatically allows one to correct the QT interval to all other heart rates. Thus individuals with the same QT interval and the same heart rate will all have the same rate corrected QT interval. These formulae therefore assume that the heart rate adaptation of the QT interval is the same in all individuals. However, it is known that QT interval rate adaptation can be influenced by disease and by drug treatment, and furthermore it is likely that even in normal subjects there will be variability within the normal range of QT interval rate adaptation. ${ }^{10-23}$ Thus it seems unlikely that an approach to the problem of QT interval rate adaptation that assumes a fixed QT interval to heart rate relation will be appropriate. Given this, it seems more appropriate that in correcting the QT interval for heart rate one should obtain the QT interval to heart rate relation for each and every subject, and use this relation to obtain a QT interval at a notional reference heart rate, for example at 60 beats $/ \mathrm{min}$. This approach would then allow one to make heart rate correction without having to make any assumptions about the QT-heart rate relation. The validity of this approach can be tested-first, by determining if this approach removes the dependence of QT interval on heart rate, and second, by determining if biological differences in the QT interval are maintained.

\section{Hypothesis}

A new heart rate to QT interval correction method should possess the following fundamental properties: it should maintain or accentuate biological QT interval variability; it should totally remove the dependence of the rate corrected QT interval on heart rate; and it should be applicable over a wide range of conditions with a wide range of differing autonomic states. 


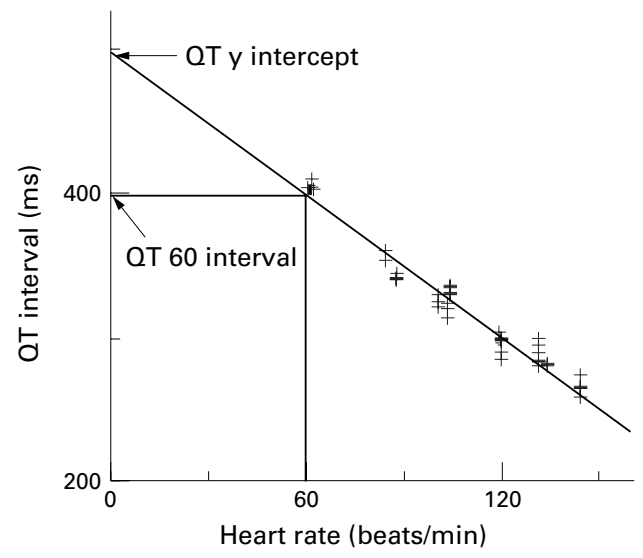

Figure 1 Example of a QT-heart rate plot obtained from an exercise test. Each point on the graph represents the mean of eight measurements of the QT interval at that heart rate. A linear regression curve is fitted to the rest and exercise QT intervals, and the curve extrapolated back to obtain values of the $O T$ interval at 60 beats/min, termed the $Q T_{60}$ interval, and at a notional heart rate of 0 beats/min, termed the QT y intercept value.

\section{Methods}

Subjects expected to have different QT intervals and different QT-heart rate slope relations were recruited, after informed consent, and with local ethics committee approval. ECG leads were attached (Marquette CASE 15).

QT INTERVAL MEASUREMENTS

QT interval from standard lead II was measured using a magnifying graticule. Each QT interval was measured five times and at least eight QT intervals, and often more, were obtained at each heart rate - that is, at least 40 QT interval measurements were made at each heart rate. ${ }^{24}{ }^{25}$ The definition of the end point of the QT interval was where the $\mathrm{T}$ wave met the isoelectric line. If this point was not clearly defined, extrapolation of the slope of the $T$ wave to the isoelectric line was used.

QT INTERVAL-HEART RATE ADAPTATION QT interval was measured at rest and during an exercise protocol to obtain a QT intervalheart rate plot for each individual. The exercise protocol consisted of a incremental bicycle exercise test with $25 \mathrm{~W}$ steps in workload occurring every four minutes. Patients exercised to exhaustion. QT interval measurements were made at rest, and then in the last minute of each workload to allow for QT interval

Table 1 Patient characteristics

\begin{tabular}{lccc}
\hline & Control & Hypertrophy & Heart failure \\
\hline Number & 16 & 16 & 34 \\
Ischaemic heart disease & $10(62 \%)$ & $1(6 \%)^{\star}$ & $19(56 \%) \dagger$ \\
Valvar heart disease & 0 & $4(25 \%)^{\star}$ & $2(6 \%)$ \\
LVID $_{\text {Diastole }}(\mathrm{mm})$ & $47(1)$ & $56(4)$ & $63(2)^{\star}$ \\
$\mathrm{LVID}_{\text {Systole }}(\mathrm{mm})$ & $29(2)$ & $35(4)$ & $51(2)^{\star} \dagger$ \\
Fractional shortening & $0.36(0.03)$ & $0.39(0.02)$ & $0.21(0.02)^{\star} \dagger$ \\
$\mathrm{LV} \mathrm{mass}_{\text {mas }}(\mathrm{g})$ & $198(15)$ & $420(48)^{\star}$ & $403(14)^{\star}$ \\
$\mathrm{HR}_{\text {rest }}($ beats/min$)$ & $74(3)$ & $68(4)$ & $86(3)^{\star} \dagger$ \\
$\mathrm{QT}_{\text {rest }}(\mathrm{ms})$ & $381(6)$ & $399(9)$ & $400(11)$ \\
$\mathrm{QT}_{\text {rest }}(\mathrm{ms}$ & $421(6)$ & $420(6)$ & $471(10)^{\star} \dagger$ \\
$\mathrm{HR}_{\text {peak exerise }}($ beats/min$)$ & $141(5)$ & $131(7)$ & $131(4)$ \\
$\mathrm{QT}_{\text {peak exercise }}(\mathrm{ms})$ & $281(7)$ & $296(11)$ & $303(10)$ \\
\hline
\end{tabular}

Values are mean (SEM) unless stated.

${ }^{\star} \mathrm{p}<0.05 v$ control; $\mathrm{tp}<0.05 v$ hypertrophy.

$\mathrm{HR}$, heart rate; LV, left ventricular; LVID, left ventricular internal dimension. hysteresis. ${ }^{26}$ In addition QT interval was measured at peak exercise.

The QT-heart rate slope relation was determined by fitting a least squares algorithm (Statview SE + graphics, version 1.03 for Apple Macintosh, from Abacus Concepts, Berkeley, California, USA) to a linear equation. The slope and position of the linear equation was thus obtained, and the line obtained was then extrapolated back to a notional heart rate of 60 beats/min, where the corresponding QT interval, termed the $\mathrm{QT}_{60}$ interval, was obtained. In addition this equation was back calculated to a notional heart rate of 0 beats/min - that is, the intercept of this line with the y axis, to obtain a value termed the QT y intercept. An example of such a calculation is shown in fig 1 .

The fit of the QT-heart rate slope obtained from application of a linear regression equation to the QT interval-heart rate data obtained during the exercise test was determined by linear regression analysis and was better than $r^{2}=0.9$ in all cases, implying that it was appropriate to use a linear equation in the range of heart rates studied. The mean (SEM) of the correlation coefficients $\left(r^{2}\right)$ was $0.93(0.02)$ for the control population, 0.95 (0.01) for the hypertrophy population, and $0.92(0.01)$ for the heart failure population (NS by analysis of variance between the three groups).

Data are presented as mean (SEM).

\section{Results}

Sixty six subjects were recruited, 34 with heart failure, 16 with left ventricular hypertrophy, and 16 controls (table 1). Some subjects had ischaemic heart disease, though all ischaemia had been fully treated either by percutaneous transluminal coronary angioplasty or by coronary artery bypass grafting. No subject was on any drug known to affect the QT interval. At rest the QT interval was the same in all three groups, but the QTc interval-using Bazett's rate correction method-was prolonged in the heart failure group owing to a high resting heart rate compared with either the left ventricular hypertrophy group or the control population (table 1).

These data therefore show that using Bazett's rate correction of the QT interval, that QT interval prolongation is found in heart failure - that is, Bazett's correction does expose biological differences in the QT interval. However, as seen in fig 2, Bazett's correction leads to an overcorrection at high heart rates - that is, Bazett's correction fails to remove the dependence of QT interval on heart rate.

The $\mathrm{QT}_{60}$ interval was obtained from the exercise test data (table 2). The QT-heart rate slope was steeper in the heart failure group than in either the control or in the hypertrophy groups by some $50 \%(\mathrm{p}<0.05)$. The QT-heart rate slope did not differ according to the aetiology of the left ventricular hypertrophy or heart failure. $\mathrm{QT}_{60}$ was prolonged in heart failure compared with either the control or the hypertrophy population, as was the QT y intercept measure of QT interval - that is, QT intervals obtained in this fashion could be corrected 


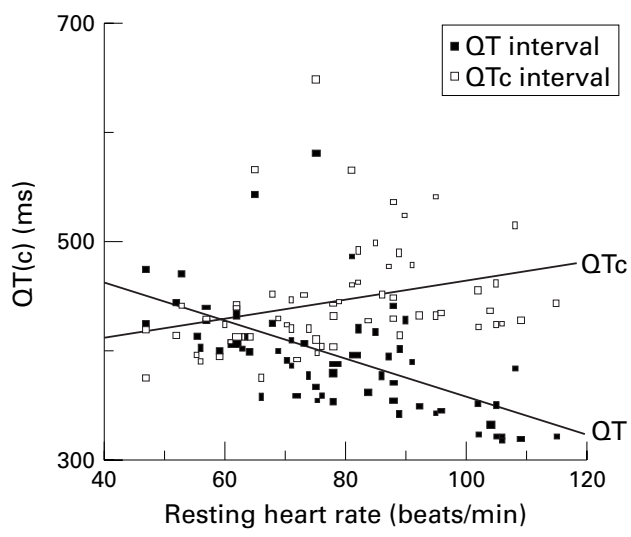

Figure 2 QT and QTc data. Data for the entire population are plotted here, before and after rate correction using Bazett's method. Each point represents the mean of the QT intervals for one subject while at rest. Linear regression analysis shows that uncorrected $Q T$ interval is significantly related to heart rate, with $r^{2}=0.34$ $(p<0.0001)$. After rate correction, however, there is still a relation with heart rate which, while weaker than that between the uncorrected $Q T$ interval and heart rate, is still significant, with $r^{2}=0.10(p<0.02)$.

Table $2 \quad Q T_{60}$ data

\begin{tabular}{llll}
\hline & Control & Hypertrophy & Heart failure \\
\hline QT $_{60}(\mathrm{~ms})$ & $404(5)$ & $411(6)$ & $454(11)^{\star} \dagger$ \\
QT y intercept (ms) $^{*}$ & $497(7)$ & $511(12)$ & $570(27)^{\star} \dagger$ \\
\hline
\end{tabular}

Values are mean (SEM).

${ }^{\star} \mathrm{p}<0.05 v$ control; $\uparrow \mathrm{p}<0.05 v$ hypertrophy.

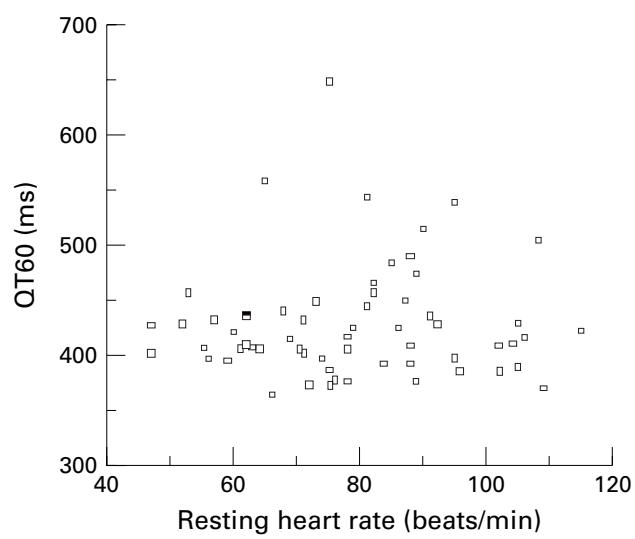

Figure $3 Q T_{60}$ plotted against heart rate. There was no relation between $Q T_{60}$ and heart rate, with $r^{2}=0.002$ $(p=0.74)$.

to any heart rate between 0 and 60 beats $/ \mathrm{min}$ and still show QT interval prolongation in heart failure.

To determine if the $\mathrm{QT}_{60}$ interval was related to heart rate, a plot of $\mathrm{QT}_{60}$ against heart rate was made (fig 3). Linear regression analysis showed that there was now no significant relation between $\mathrm{QT}_{60}$ interval and heart rate - that is, the $\mathrm{QT}{ }_{60}$ method of QT interval heart rate correction has totally removed the dependence of QT interval on heart rate. This absence of relation between heart rate and QT interval was also found when the QT y intercept measure was used $\left(r^{2}=0.005, \mathrm{p}=0.60\right)$.

\section{Discussion}

There have been many formulae proposed to correct the QT interval for the effects of varying heart rate. ${ }^{1-9} 27$ Four of these propose that the relation between QT interval and RR interval is linear. ${ }^{47}$ Two propose linear QT-heart rate relations, and in one the formula is derived from fundamental thermodynamic principles. $^{927}$ The other formulae propose more complex relations between QT interval and heart rate. Three of these formulae accept that there are important differences based on sex or age between individuals in the dependence of the QT interval on heart rate. ${ }^{3}{ }^{4}$ None of the proposed formulae, however, incorporate differences based on disease status. As many cardiac diseases alter both the QT interval and the function of the autonomic nervous system, it would not be surprising if the rate dependence of the QT interval was altered in heart disease. It is in just these individuals with diseased hearts that one wishes to measure the QT interval. Thus in order to use a formula to correct the QT interval in patients with heart disease, one will need a very large dataset that incorporates the effect of the heart disease as well as the effects of cardioactive drugs on the rate dependence of the QT interval. This approach may not be practical.

An alternative approach is to determine the rate dependence of the QT interval for each individual, and use this to correct the QT interval to a notional heart rate. The data presented here show that using individualised heart rate dependence for the QT interval allows one to correct the QT interval for the effects of heart rate. Furthermore, the results show that using exercise test data to correct the QT interval individually to a nominal heart rate of 60 beats/min satisfies the two important criteria of a QT-heart rate correction algorithm, which are that the rate correction method should totally remove the dependence of QT interval on heart rate but at the same time maintain biological differences.

Given that it is physiologically based-and therefore makes no assumptions about the nature of the relation between QT interval and heart rate - and that it completely removes all dependence on heart rate while at the same time maintaining biological diversity, this method of QT interval-heart rate correction is superior to previous methods that have assumed a fixed relation between QT interval and heart rate, as those methods failed to remove the relation between corrected QT interval and heart rate. Because Bazett's method of correcting for heart rate consistently overcorrected the QT interval at high heart rates, and because high resting heart rates are found in heart failure, there has up to now been some concern that prolongation of the QTC interval found in heart failure is not the result of a genuine prolongation of repolarisation but is rather an artefact of the rate correction formulae. The findings from this study indicate that there is genuine prolongation of repolarisation in heart failure. This finding may aid our understanding of arrhythmogenesis in heart failure. Lethal ventricular arrhythmias are particularly prevalent in heart failure, as is $\mathrm{QT}_{60}$ interval prolongation. It remains to be seen whether $\mathrm{QT}_{60}$ prolongation relates to arrhythmogenesis, but if it does, this may suggest that 
an important component of sudden cardiac death in heart failure relates to mechanisms dependent upon prolonged repolarisation, and these particularly include repetitive afterdepolarisations. ${ }^{28} 29$

Though for reasons of convention I arbitrarily chose to correct the QT interval back to a notional heart rate of 60 beats/min, the fact that the QT y intercept - which is when the notional heart rate was 0 beats/min-showed no relation with heart rate and was prolonged in heart failure meant that we could have chosen any value of heart rate between 0 and 60 beats/min for the notional heart rate corrected QT interval.

\section{IMPLICATIONS}

This study may have important implications for future QT interval analysis, in particular of data obtained from 24 hour Holter ambulatory recorders. Computer software that can determine QT interval from Holter recorders is becoming increasingly widespread. This approach to QT interval analysis may have many advantages over more conventional ways of measuring the QT interval. Not only should this approach minimise variability in the measurement of the QT interval, which may allow the QT interval to be used more reliably in predicting sudden cardiac death, but it will also allow us to analyse data in novel ways. With the large number of QT-heart rate points one can obtain-approximately 100000 QT intervals in 24 hours - one could calculate the QT-heart rate relation on, say, an hour to hour basis over the 24 hours, and use this to calculate an hourly $\mathrm{QT}_{60}$ interval, which can then be tested against variables of interest. This looks like a promising approach to correcting the QT interval for heart rate without the need to make any assumptions about the relation between QT interval and heart rate; however, this physiologically based method of correction of the QT interval will need to be validated for day to day exercise, as opposed to laboratory based exercise tests, before it can be held to be generally applicable.

\section{LIMITATIONS}

This method of correcting the QT interval for the effects of heart rate requires a number of measurements of QT interval at different heart rates, which in this study were obtained from an exercise test. This is clearly a time consuming exercise, and it may be that this method will be principally limited to the research field. However, QT interval measurement is of most interest in patients with heart disease, many of whom undergo exercise testing. With the increasing use of computer based QT interval analysis, it may be relatively easy to incorporate this method of analysis into future exercise machines.
The department receives a grant from the British Heart Foundation

1 Bazett HC. An analysis of the time-relations of electrocardiograms. Heart 1920;7:353-70.

2 Fridericia LS. Die Sytolendauer im Elektrokardiogramm bei normalen Menschen und bei Herzkranken. Acta Med Scand 1920;53:460-9.

3 Ashman R. The normal duration of the QT interval. Am Heart f 1942;23:520-2

4 Adams W. The normal duration of the electrocardiographic ventricular complex. 7 Clin Invest 1936;15:330-5.

5 Ljung O. A simple formula for clinical interpretation of the QT interval. Acta Med Scand 1949;134:70-9.

6 Schlamowitz I. An analysis of the time relationships within the cardiac cycle in electrocardiograms of normal men I. The duration of the QT interval and its relationship to the cycle length. Am Heart f 1946;31:320-9.

7 Simonson E, Cady LD, Woodbury M. The normal QT Simonson E, Cady LD, Woodbury
interval. Am Heart 7 1962;63:740-7.

8 Sarma JS, Sarma RJ, Bilitch M, et al. An exponential formula for heart rate dependence of QT interval during exercise and cardiac pacing in humans: reevaluation of Bazett's formula. Am f Cardiol 1984;54:103-8.

9 Kovacs SJ. The duration of the QT interval as a function of heart rate: a derivation based on physical principles and a comparison to measured values. Am Heart f 1985;110: 872-8.

10 Gill JS, Baszko A, Xia R, et al. Dynamics of the QT interval in patients with exercise-induced ventricular tachycardia in normal and abnormal hearts. Am Heart f 1993;126:135763.

11 Cuomo S, De Caprio L, Acanfora D, et al. Relationship between QT interval duration and exercise-induced ventricular arrhythmias. Eur Heart f 1989;10:622-7.

12 Funck-Brentano C, Kibleur Y, Le Coz F, et al. Rate dependence of sotalol-induced prolongation of ventricular repolarization during exercise in humans. Circulation 1991;83: 1arization

13 Sarma JS, Venkataraman K, Samant DR, et al. Effect of propranolol on the QT intervals of normal individuals during exercise: a new method for studying interventions. Br Heart 1988:60:434 9 .

14 Algra A, Roelandt JRTC, Tijssen JGP, et al. Effect of $\beta$-blockers on the relation between QT-interval and heart rate in exercise ECG. Eur Heart F 1987;8:71-4.

5 Viitasalo M, Karjalainen J. QT intervals at heart rates from 50 to 120 beats per minute during 24-hour electrocardiographic recordings in 100 healthy men. Effects of atenolol. Circulation 1992;86:1439-42.

16 Alexopoulos D, Rynkiewicz A, Yusuf S, et al. Diurnal variations of QT interval after cardiac transplantation. Am $\mathcal{F}$ Cardiol 1988;61:482-5.

17 Ewing DJ, Boland O, Neilson JM, et al. Autonomic neuropathy, QT interval lengthening, and unexpected deaths in male diabetic patients. Diabetologia 1991;34:1825 .

18 Kahn JK, Sisson JC, Vinik AI. QT interval prolongation and sudden cardiac death in diabetic autonomic neuropathy. $\mathcal{F}$ Clin Endocrinol Metab 1987;64:751-4.

19 Kadish AH, Weisman HF, Veltri EP, et al. Paradoxical effects of exercise on the QT interval in patients with polymorphic ventricular tachycardia receiving type Ia antiarrhythmic agents. Circulation 1990;81:14-19.

20 Sadeh D, Shannon DC, Abboud S, et al. Altered cardiac repolarization in some victims of sudden infant death syndrome. $N$ Engl F Med 1987;317:1501-5.

21 Romano M, Di Maro T, Carella G, et al. Relation between heart rate and OT interval in exercise-induced myocardial ischemia. Am 7 Cardiol 1985;56:861-2.

22 Gardner MJ, McCarthy DM, Josephson ME. Effect of myocardial ischemia and type I antiarrhythmic drug therapy on exercise QT intervals. Can f Cardiol 1985;1:37-40.

23 Juul-Muller S. Corrected QT interval during one year follow-up after an acute myocardial infarction. Eur Heart $\mathcal{f}$ 1986;7:299-304

24 Campbell Cowan J, Yussoff $\mathrm{K}$, Moore M, et al. Importance of lead selection in QT interval measurement. Am Heart $\mathcal{F}$ 1988;61:83-7.

25 Ahnve S. Errors in the visual determination of corrected QT QTc) interval during acute myocardial infarction. $\mathcal{F} \mathrm{Am}$ Coll Cardiol 1985;5:699-702.

26 Lau CP, Freedman AR, Fleming S, et al. Hysteresis of the ventricular paced QT interval in response to abrupt changes in pacing rate. Cardiovasc Res 1988;22:67-72.

27 Hodges M, Salerno D, Erlein D. Bazett's QT correction reviewed: evidence that a linear QT correction for heart rate is better [abstract]. I Am Coll Cardiol 1983;1:694.

28 January CT, Riddle JM. Early after depolarisations: mechanisms of induction and block. Circ Res 1989;64:977-90.

29 January CT, Makielski JC. Triggered arrhythmias: new insights into basic mechanisms. Curr Opin Cardiol 1990;5: $65-8$. 